\title{
The Second Neurocritical Care Research Conference
}

\author{
Jose I. Suarez ${ }^{1}$ Peter D. LeRoux ${ }^{2}$
}

Published online: 2 April 2015

(c) Springer Science+Business Media New York 2015

One of the missions of the Neurocritical Care Society (NCS) is to foster clinical, experimental, and outcomes research focused on developing innovative and cost-effective medical and surgical interventions for acute neurological disorders. To achieve this mission, NCS created a research committee. This committee organized the Neurocritical Care Research Conference. The most important outcome of the First Neurocritical Care Research Conference (September 2009) was the creation of the Neurocritical Care Research Network (NCRN) [1]. Following the success of that first endeavor, the Second Neurocritical Care Research Conference took place in Houston, TX May 18-20, 2012. This symposium was funded by NINDS (1R13NS077610-01, P.I. Jose I. Suarez), Baylor St. Luke's Medical Center (Neuroscience Center) in Houston, and the Integra Foundation and endorsed by the Baylor College of Medicine (BCM) and the NCS.

The overall goal of this interdisciplinary scientific gathering was to bring together a diverse group of academic scientists and physicians with a common interest in understanding and advancing research in multimodality monitoring in neurocritical care diseases, in large part because monitoring patient disease and care is a fundamental aspect of neurocritical care. The need for the design of adequate clinical studies to test these monitoring

Jose I. Suarez

jisuarez@bcm.edu

1 Division of Vascular Neurology and Neurocritical Care, Department of Neurology, Baylor College of Medicine, Houston, TX, USA

2 Lankenau Medical Center, Thomas Jefferson University, Philadelphia, PA, USA techniques was addressed in the context of the recently created NCRN to facilitate collaboration and patient enrollment. In addition, this conference provided a unique opportunity for trainees, junior faculty, and other scientists to interact with investigators in the field of neurocritical care. The conference opened with a discussion on new approaches to multifaceted therapeutic bundles in neurocritical care followed by five scientific sessions: (1) Global Brain Monitoring in the Neurocritical Care Unit; (2) Regional Brain Monitoring in the Neurocritical Care Unit; (3) Data Collection and Interpretation; (4) Conventional Clinical Trial Design of Multimodality Monitoring; and (5) Alternative Clinical Trial Design.

The meeting had 158 registrants and 135 attendees. This represents a $26 \%$ increase in attendance compared to our first research conference. Attendees came from all parts of the world including the United States, Canada, Latin America, Europe, Japan, India, and the Middle East. We had lively and interesting discussions throughout the meeting. The conference was successful and well received with $94 \%$ stating that it was fair and balanced. At the end of the meeting, there was consensus on two major points: (1) we need a common language to describe the technology available and the information it provides to pursue further research to advance the field and (2) technology alone will not impact upon clinical outcome without specific interventions and pathways that can modify and ameliorate the biochemical cascade unleashed by brain insults. Two projects have since evolved from the Second Neurocritical Care Research Conference: (1) the International Multidisciplinary Consensus Conference on Multimodality Monitoring in Neurocritical Care, a collaboration between the NCS, the European Society of Intensive Care Medicine, the Society of Critical Care Medicine, and the Latin 
American Brain Injury Consortium [2] and (2) the Common Data Elements project in subarachnoid hemorrhage in collaboration with the Subarachnoid Hemorrhage International Trialists Collaboration [3].

This issue of Neurocritical Care contains the summary statements that were prepared based on the presentations and discussions that followed during this Second Neurocritical Care Research Conference. Similar to the first conference, the content of each manuscript reflects input from conference organizers, speakers, session moderators, and attendees. We have prepared five summary statements that mirror each of the sessions. In addition, we have listed the names and academic affiliations of all investigators who attended the meeting at the end of each manuscript. We are very grateful to all of them for their participation and enthusiasm.

Acknowledgments The Second Neurocritical Care Research Conference was funded by the National Institutes of Health (NINDS) (R13 NS077610, PI: Suarez JI), the Integra Foundation, and the Baylor St Luke's Medical Center in Houston, TX.
Disclosures The content is solely the responsibility of the authors and does not necessarily represent the official views of the National Institute of Neurological Disorders and Stroke of the National Institutes of Health.

\section{References}

1. Suarez JI, Geocadin R, Hall C, Le Roux P, Smirnakis S, Wijman CAC, Zaidat OO, for the First Neurocritical Care Research Conference Investigators. The Neurocritical Care Research Network: NCRN. Neurocrit Care. 2012;16(1):29-34.

2. Le Roux P, Menon DK, Citerio G, Vespa P, Bader MK, Brophy $\mathrm{GM}$, et al. Consensus Summary Statement of the International Multidisciplinary Consensus Conference on Multimodality Monitoring in Neurocritical Care: a statement for healthcare professionals from the Neurocritical Care Society and the European Society of Intensive Care Medicine. Neurocrit Care. 2014;21:297-361.

3. Macdonald RL, Cusimano MD, Etminan N, Hanggi D, Hasan D, Ilodigwe D, et al. Subarachnoid Hemorrhage International Trialists Data Repository (SAHIT). World Neurosurg. 2013;79(3-4):41822. 